COMPARISON OF EXTRACTION AND BEAM TRANSPORT SIMULATIONS WITH EMITTANCE MEASUREMENTS FROM THE ECR ION SOURCE VENUS

D. Winklehner, D. Todd, J. Benitez, M.

Strohmeier, D. Grote, D. Leitner

November 12, 2010

Journal of Instrumentation 
This document was prepared as an account of work sponsored by an agency of the United States government. Neither the United States government nor Lawrence Livermore National Security, LLC, nor any of their employees makes any warranty, expressed or implied, or assumes any legal liability or responsibility for the accuracy, completeness, or usefulness of any information, apparatus, product, or process disclosed, or represents that its use would not infringe privately owned rights. Reference herein to any specific commercial product, process, or service by trade name, trademark, manufacturer, or otherwise does not necessarily constitute or imply its endorsement, recommendation, or favoring by the United States government or Lawrence Livermore National Security, LLC. The views and opinions of authors expressed herein do not necessarily state or reflect those of the United States government or Lawrence Livermore National Security, LLC, and shall not be used for advertising or product endorsement purposes. 


\title{
COMPARISON OF EXTRACTION AND BEAM TRANSPORT SIMULATIONS WITH EMITTANCE MEASUREMENTS FROM THE ECR ION SOURCE VENUS*
}

\author{
D. Winklehner", D. Todd, J. Benitez, M. Strohmeier, LBNL, Berkeley, CA 94704, USA \\ D. Grote, LLNL, Livermore, CA 94551, USA \\ D. Leitner, LBNL, Berkeley, CA 94704, MSU, East Lansing, MI 48824, USA
}

\begin{abstract}
The versatility of ECR (Electron Cyclotron Resonance) ion sources makes them the injector of choice for many heavy ion accelerators. However, the design of the LEBT (Low Energy Beam Transport) systems for these devices is challenging, because it has to be matched for a wide variety of ions. In addition, due to the magnetic confinement fields, the ion density distribution across the extraction aperture is inhomogeneous and charge state dependent. In addition, the ion beam is extracted from a region of high axial magnetic field, which adds a rotational component to the beam. In this paper the development of a simulation model (in particular the initial conditions at the extraction aperture) for ECR ion source beams is described. Extraction from the plasma and transport through the beam line are then simulated with the particle-in-cell code WARP. Simulations of the multispecies beam containing Uranium ions of charge state $18+$ to $42+$ and oxygen ions extracted from the VENUS ECR ion source are presented and compared to experimentally obtained emittance values.
\end{abstract}

\section{INTRODUCTION}

The superconducting Versatile ECR ion source for

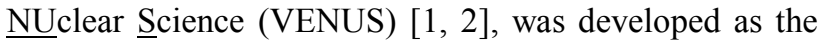
prototype injector for the Facility for Rare Isotope Beams (FRIB) and as injector ion source for the 88-Inch Cyclotron at Lawrence Berkeley National Laboratory [3, 4]. Like most ECR ion sources VENUS operates in a minimum $\mathrm{B}$ field configuration which means that a magnetic sextupolar field for radial confinement is superimposed with a magnetic mirror field for axial confinement. Consequently:

- Ions are extracted out of a region with high axial magnetic field (in VENUS typically $2 \mathrm{~T}$ ) which then continuously decreases as the ions move along in axial direction, adding a rotational component to the beam.

- Due to the sextupolar field, the total magnetic field inside the source is not rotationally symmetric and thus the spatial distribution of the ions at extraction resembles a triangle rather than a circle. This is also confirmed by beam imaging of single- or fewspecies ion beams (e.g. Figure 1) [4].

Furthermore, the extracted beam often consists of more

\footnotetext{
* This Research was conducted at LBNL and was supported by the U.S. Department of Energy under Contract DE-AC02-05CH11231.

\# winklehner@frib.msu.edu
}

than 30 different ion species with different mass-tocharge ratios which makes modeling even more complicated. At the moment, several groups are developing versatile extraction codes that are able to handle the complex initial conditions presented by ECR ion source plasmas. The main goals are:

- To better understand the underlying plasma physics that leads to these initial conditions.

- To create a design tool for future ECR extraction systems.

The work described here represents the current status of a long-term effort to create a highly adaptable, advanced simulation code utilizing the well-established PIC (particle-in-cell) code WARP [5].

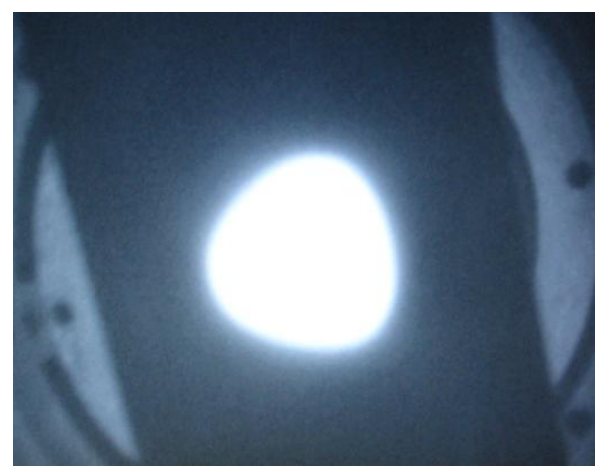

Figure 1: Image of a triangular $\mathrm{He}^{+}$beam $80 \mathrm{~cm}$ after extraction. Taken with a Tantalum viewing screen (heating up locally upon ion impact) and a camera [4].

Many of the issues regarding the extraction simulation and the beam transport through the beam line have been addressed in earlier work by D. Todd et al. $[4,6]$ and will be reviewed briefly in the following sections. Results of a Uranium beam simulation using an efficient way to obtain the initial conditions will then be presented and compared to emittance measurements.

\section{SIMULATIONS}

The simulation of a multispecies ion beam from plasma extraction to the position of the diagnostics box can be divided into three separate simulations (see Figure 2), each employing different simulation methods which are discussed in the following subsections. 


\section{Initial Conditions}

(See Figure 2, region I: Inside the plasma) The initial particle spatial- and velocity-distributions of each species on the plasma side of the extraction simulation are a vital part of any extraction simulation. These initial conditions are not known for ECR plasmas and are subject to debate. Different approaches exist, including:

- Considering effects within the plasma such as negative potentials forming along the axis which would increase the density of highly charged ions near the axis $[7,8]$

- Requiring the ions to be created at points inside the source which a.) exhibit higher or equal magnetic flux density than the extraction region and are b.) connected to the aperture via a field line, along which the ions can travel [9].

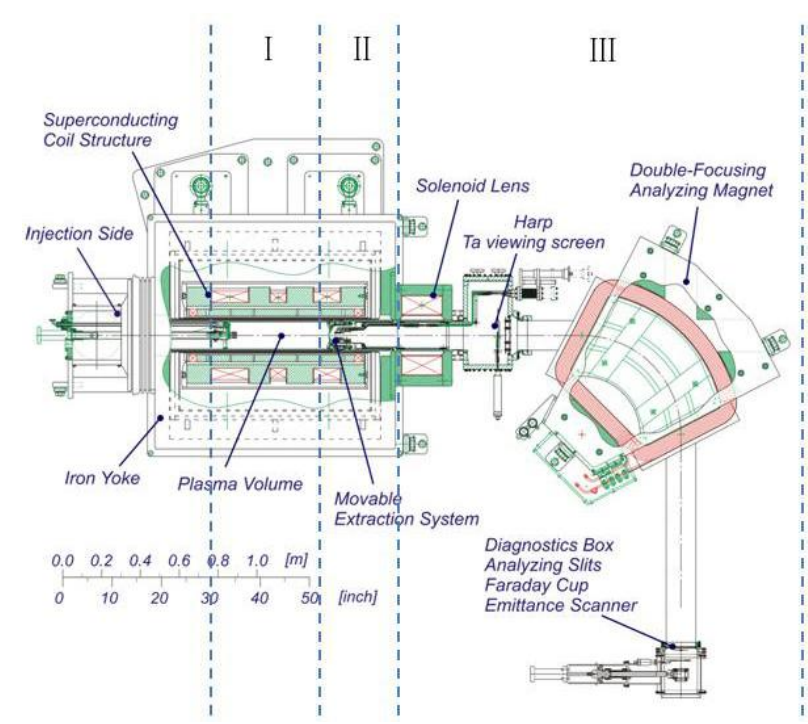

Figure 2: Layout of VENUS and its beam transport system. Initial conditions, extraction simulation and beam line transport correspond to regions I, II and III respectively.

For this work, a semi-empirical approach was taken: VENUS, similar to most ECR ion sources, operates with a negatively biased metal plate (biased disk) on the injection side of the source to enhance the flux of low energy electrons into the plasma. The biased disk is kept at a voltage of $-50 \mathrm{~V}$ to $-100 \mathrm{~V}$, thus providing the ions with enough kinetic energy to sputter the surface. As seen in Figure 3, upper left, this disk exhibits two kinds of sputter-marks:

- A large triangular discoloration: Plasma marks which match the magnetic field lines crossing the ECR resonance zone.

- A smaller triangle of about $4 \mathrm{~mm}$ height that is etched deeply into the disk and has distinct edges.

The same discoloration can be found on the extraction plate (see Figure 3, upper right). From the size of the bright triangle on the extraction plate which fills the extraction aperture completely, one could presume that ions are extracted evenly distributed over the whole surface of the aperture. This, however, contradicts the triangular beam cross-section image seen in Figure 1 as well as emittance measurements indicating that higher charge states are emitted from a "virtual" extraction aperture of smaller size than the actual one [10]. Clearly, requiring ions to be created on the ECR resonance surface and following field lines to the extraction aperture alone is not enough.
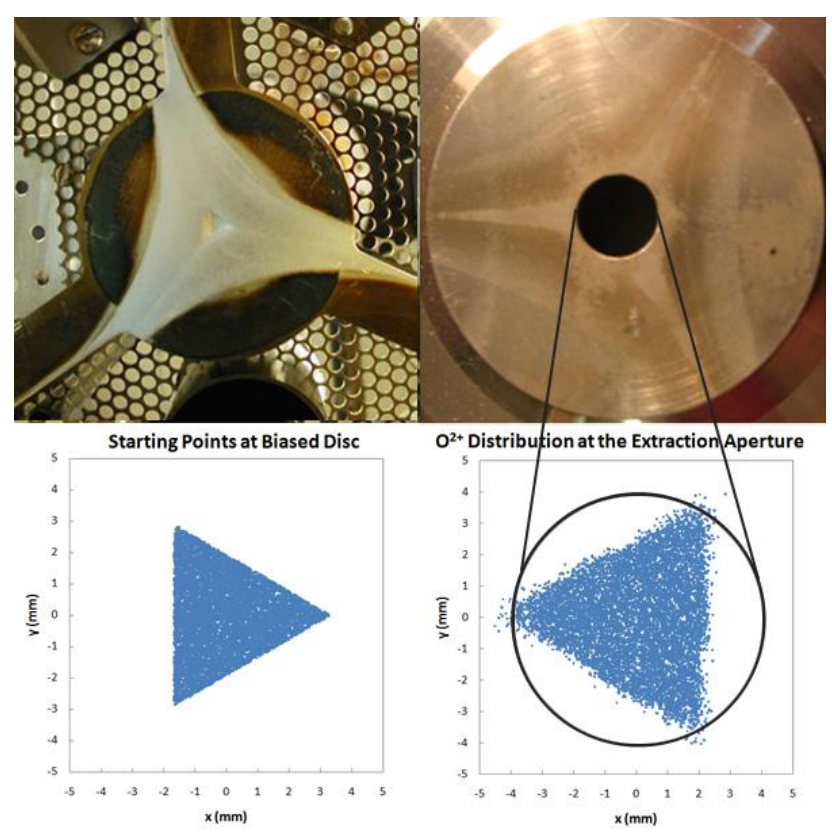

Figure 3: Sputter marks on biased disk (upper left), marks on extraction aperture (upper right), initial particle distribution on biased disk matching sputter marks (lower left) and particle spatial distribution after tracking through the source, shortly before extraction aperture (lower right).

Therefore the small sputtered triangle on the biased disk was examined further. The small triangle's depth and sharp edges indicate a higher ion density near the axis and also give rise to the assumption that collisions which cause radial diffusion must be a secondary effect between the resonance zone and the biased disk [6]. Because the ions are cold $(\mathrm{a}$ few $\mathrm{eV}$ ) resulting in small Larmor radii it is reasonable to assume that they mainly follow the magnetic field lines. In addition, there is no reason why the direction towards the biased disk should be preferred, thus it can be argued that a similar ion beam distribution can be found on the extraction side of the source.

The initial (and direct) approach for producing the starting conditions for the sheath extraction simulation was the following: Ions are randomly distributed on a triangle corresponding to the sputtered triangle and given a random velocity corresponding to a Boltzmann distribution with a peak temperature of $2 \mathrm{eV}$. Subsequently, these ions are tracked through the magnetic field of the source 
to the extraction aperture by means of a Leapfrog algorithm. Collisions are neglected assuming low plasma density and small Larmor radii. However, obtaining a statistically relevant number of $\sim 10000$ ions per species $(\sim 30)$ is a very time-consuming process and so an alternative (faster) method has been developed.

In this alternate method, particles are still initialized the same way, but instead of tracking them through the source, each particle's respective gyro-motion guidingcentre is calculated and only the field-line originating at that point is tracked through the source. At the respective end-point, an appropriately scaled Larmor radius is applied and the particle is put on a random point on a circle with this scaled radius around the guiding-centre. Because travelling through the source's magnetic field leads to a shift from the ion's transversal velocity to the longitudinal velocity component, velocities are recalculated from the scaled Larmor-radius in the end. As an example, starting points at the biased disk as well as the derived (spatial) initial conditions at the extraction aperture for $\mathrm{O}^{2+}$ can be seen in the lower part of Figure 3. Testing both methods against each other with selected ion species showed very good agreement.

One of the limitations of this model for the initial conditions at the plasma interface is that it assumes the same initial spatial distribution of the ions at the bias disk for all charge states. As this assumption might not be valid for the highest charge states the model might need further refinement.

\section{Extraction Simulation}

(See Figure 2, region II: The extraction system) WARP includes both a two-dimensional, axially symmetric plasma sheath extraction model similar to IGUN [11] and a three-dimensional sheath extraction model comparable to KOBRA [12]. In order to allow for sufficient resolution at the plasma sheath while keeping the simulation time reasonable, the following two-step approach has been taken:

1. An axially symmetric beam with the same species parameters, currents and energies as the triangular beam is extracted using the two-dimensional model. The beam is tracked through the simulation several times, until a relaxation of the combined potential of applied fields and self-fields has been reached.

2. The obtained potential is stored and used as an applied field in the second step, where the beam is initialized with the obtained triangular particle distributions and the simulation is run in threedimensional mode.

Previous tests against a full 3D simulation have confirmed the validity of this approach [4].

\section{Beam line Transport Simulation}

(See Figure 2, region III: The beam line) Since the longitudinal velocity in the remaining beam line is much higher than the transverse, a two-dimensional Poisson solver can be used to simulate the beam line transport (slice mode). The longitudinal self-fields are neglected but the motion through the three dimensional analyzing magnet fields is simulated [4].

The beam-influencing components in the beam transport simulation are (see Figure 2):

- The solenoid field of the source

- The solenoid lens

- The dipole analyzing magnet

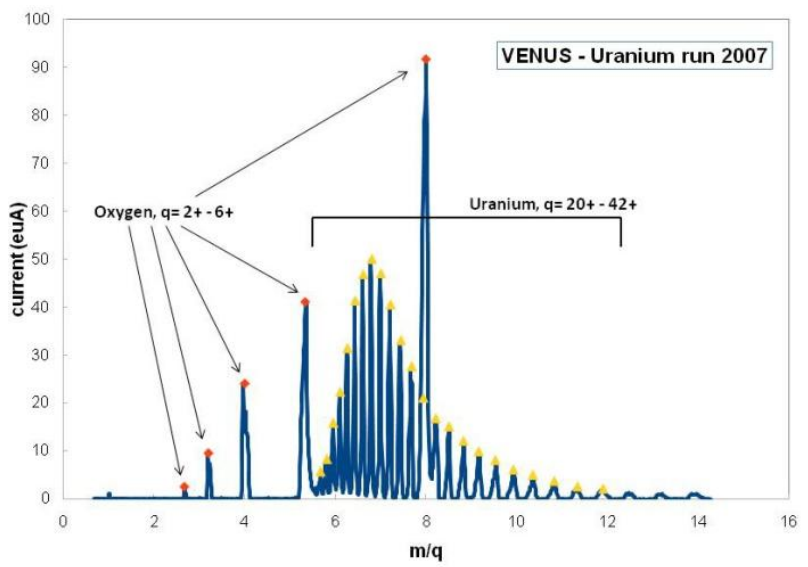

Figure 4: Spectrum of a Uranium beam from VENUS used for the initial species and current distribution in the extraction simulation.

\section{RESULTS}

One of the most important beams for FRIB are high intensity medium charge state uranium beams [13]. Uranium beam data from VENUS are thus available and benchmarking these data against simulations is of particular interest. 28 different ion species, namely Oxygen (as support gas) with charge states $2+$ to $6+$ and Uranium with charge states $20+$ to $42+$ were used in the simulations. The species distribution and respective currents in e $\mu \mathrm{A}$ were obtained from the VENUS spectrum which was optimized for $\mathrm{U}^{35+}$ as shown in Figure 4. The key initial parameters of the simulation are listed in Table 1. While source and puller voltages, drain current and magnetic fields are known, the plasma parameters such as ion temperature and plasma density have to be set by the user. Choosing these initial parameters differently can change the simulation and will have to be studied in more detail.

Table 1: Initial simulation parameters $\left(E_{k i n}\right.$ is added to the longitudinal Temperature in order to account for presheath acceleration and the current distribution from the spectrum is normalized to the total drain current)

\begin{tabular}{|l|l|}
\hline Parameter & Value \\
\hline Ion mean Temperature & $2 \mathrm{eV}$ \\
Uranium E $_{\text {kin }}$ (longitudinal) & $\sim 3 \mathrm{eV}$ \\
Electron Temperature (in sheath) & $5 \mathrm{eV}$ \\
Source Voltage & $20 \mathrm{kV}$ \\
Puller Voltage & $-2 \mathrm{kV}$ \\
Total drain current & $1.6 \mathrm{~mA}$ \\
Max. mag. flux density at extraction & $2.1 \mathrm{~T}$ \\
\hline
\end{tabular}



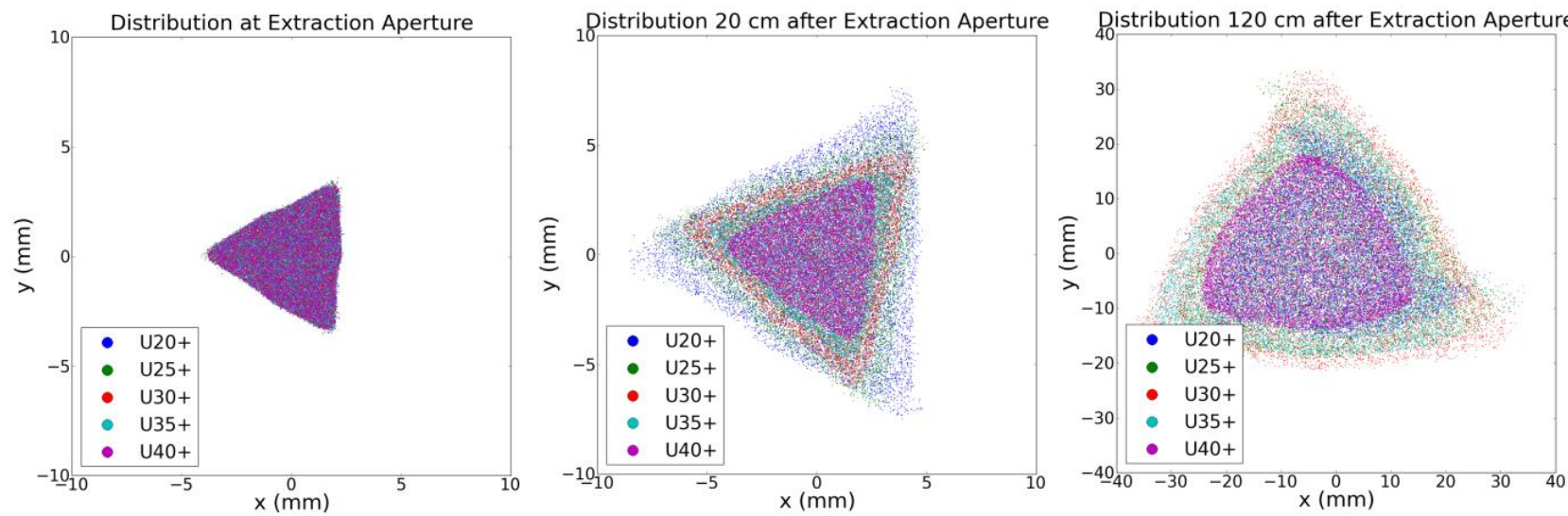

Figure 5: Spatial distributions for $U^{20+}, U^{30+}$ and $U^{40+}$ at the extraction aperture (left), $20 \mathrm{~cm}$ after (middle) and $120 \mathrm{~cm}$ after (right). Note the different scale for $x$ and $y$ in the last plot, this is the z-position right before the analyzing magnet (this simulation was done with a neutralization of $80 \%$ and the focusing magnet was set for $U^{35+}$ ).

\section{Results from Simulations}

The influence of the source solenoid on the extraction end can be seen in Figure 5. The spatial distributions of five different charge states of Uranium $(q=20+$ to $40+$ in steps of 5) are plotted before extraction and $20 \mathrm{~cm}$ after the extraction aperture (where the particles are handed over to the beam line transport simulation) as well as shortly before the dipole magnet. A noticeable difference in rotation and focusing for different charge states can be seen.

Figure 6 shows a set of horizontal beam envelopes $\left(\mathrm{U}^{35+}\right.$ selected by magnets). Different focusing of the different ion species can clearly be seen in the "Glaser" (focusing solenoid) region, especially noticeable is the over-focusing of low-m/q species which can lead to regions of high space charge density and thus formation of a hollow beam or increased emittance.

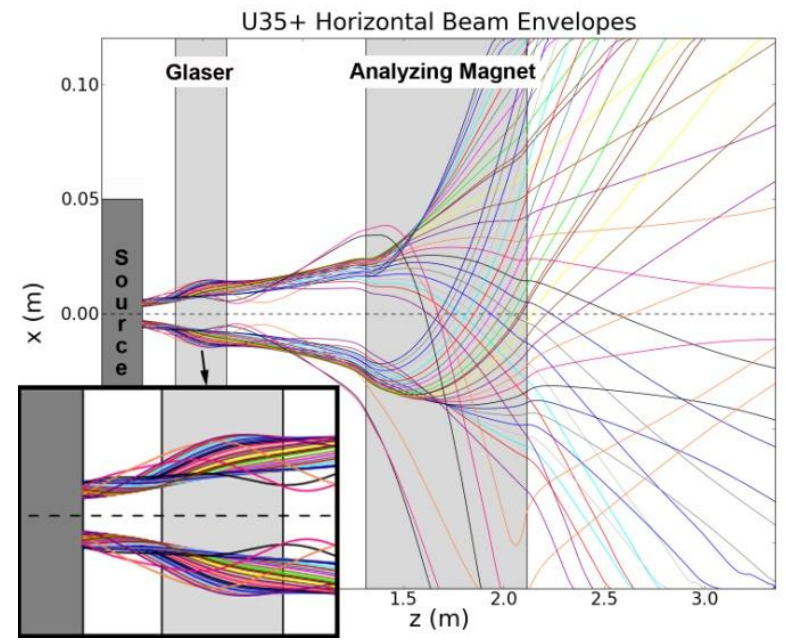

Figure 6: Horizontal beam envelopes for a $U^{35+}$ beam. The coordinates in the analyzing magnet (bend) are "warped" by the code to have a virtual, straight beam line for the selected species.

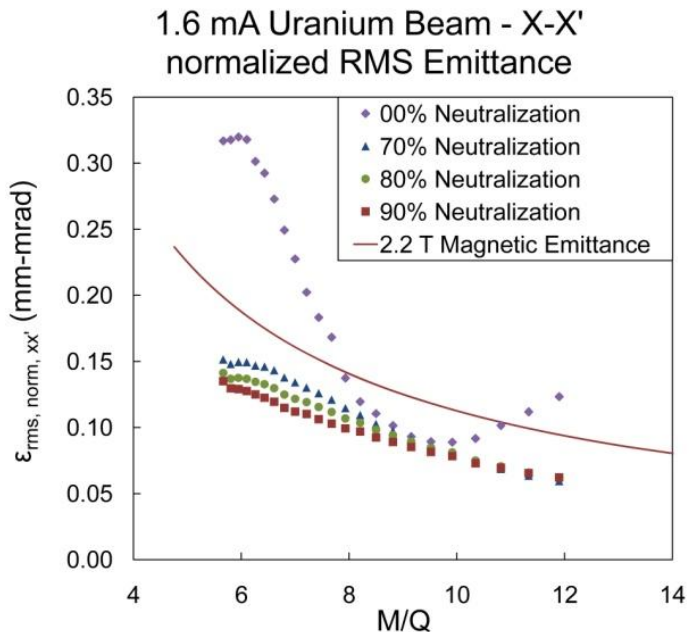

Figure 7: Comparison of horizontal ( $\left.x x^{\prime}\right)$ emittances for $U^{20+}$ to $U^{40+}$ for different neutralization factors.

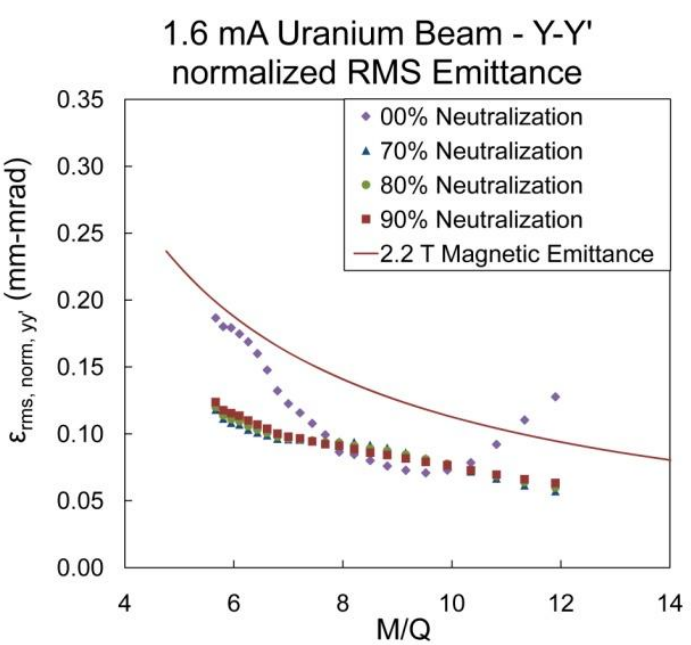

Figure 8: Comparison of vertical (yy') emittances for $U^{20+}$ to $U^{40+}$ for different neutralization factors. 
Emittances for $\mathrm{U}^{30+}$ to $\mathrm{U}^{40+}$, both horizontal $\left(\mathrm{x}-\mathrm{x}^{\prime}\right)$ and vertical (y-y'), are plotted in Figure 7 and Figure 8, respectively, for different levels of neutralization $(0 \%$, $70 \%, 80 \%$ and $90 \%$ ). The neutralization is due to rest gas ionization in the beam line and accumulation of now free electrons around the beam. In a previous work, it was experimentally observed that although the beam is not pulsed, the extracted ion beams are not fully neutralized at pressures of less than 2e-7 mbar in the beam line [14]. Therefore different neutralization levels were tested in the model by scaling the total beam current.

The emittances were obtained by evaluating the particle data from the simulations at a position $3.3 \mathrm{~m}$ after the extraction aperture as 1-rms emittances normalized to the beam energy.

The magnetic emittance displayed for comparison in Figure 7, Figure 8 and Figure 10 was calculated according to [15] by

$$
\varepsilon_{m a g}^{x x^{\prime}-r m s-n o r m}=0.032 \cdot r^{2} \cdot B_{0} \cdot \frac{1}{M / Q}
$$

with $r$ the extraction aperture radius of $4 \mathrm{~mm}$ and $\mathrm{B}_{0}$ the magnetic field on axis at the position of the extraction aperture. As expected, Figure 7 generally shows a decrease of emittance with increasing neutralization, and a strong influence of space-charge for $0 \%$ neutralization. Figure 8 shows a less pronounced change in emittance for changes from $70 \%$ to $90 \%$ in neutralization level but still a big influence for $0 \%$.

Beam cross-sections of a $\mathrm{U}^{35+}$ beam for different neutralization levels, at the position of the emittance scanners, are shown in Figure 9.

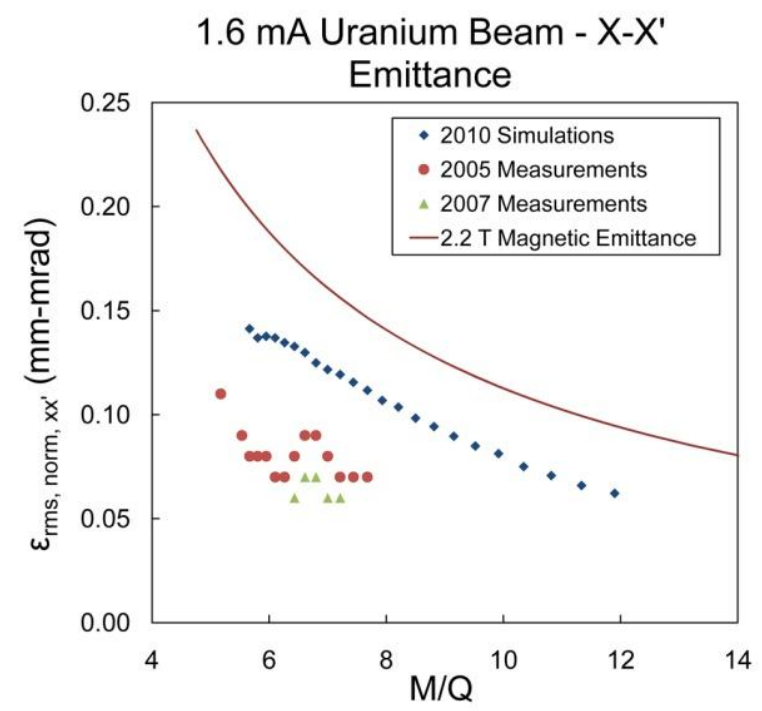

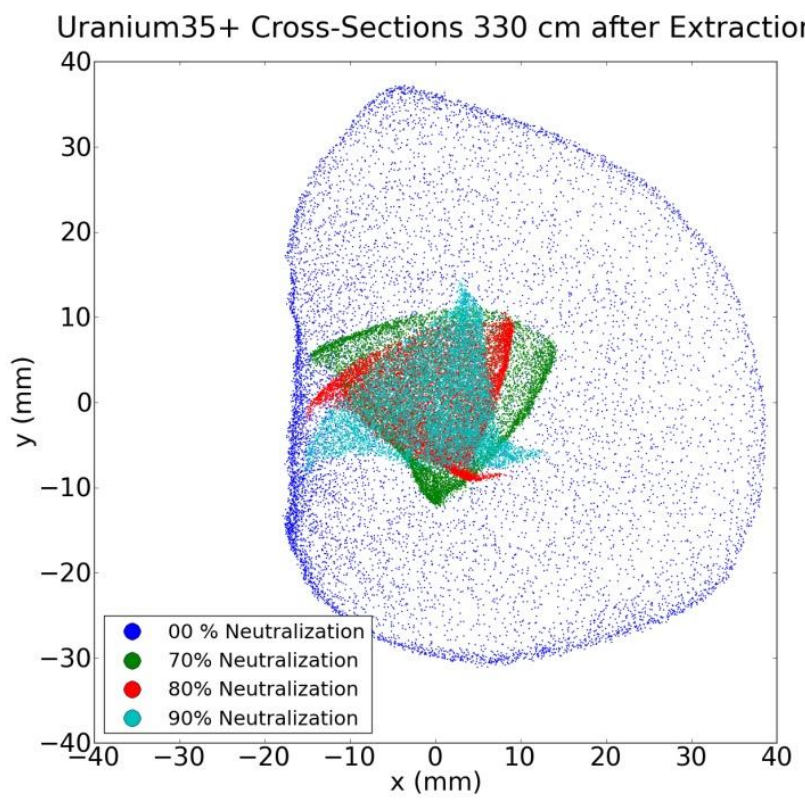

Figure 9: Comparison of $U^{35+}$ cross-sections at the diagnostics position (3.3 $\mathrm{m}$ after extraction), $1.6 \mathrm{~mA}$ total extracted current.

Two effects are noticeable:

1. Increasing beam size with decreasing neutralization level for $0 \%-80 \%$ neutralization due to the selfrepulsion of the beam.

2. Hollowing of the beam, for a neutralization level of $70 \%$ and less. This effect can be attributed to overfocusing of $\mathrm{O}^{4+}-\mathrm{O}^{6+}$; species that cannot be avoided when using Oxygen as support gas.

2-rms beam sizes in $\mathrm{x}$ and $\mathrm{y}$ direction of a $\mathrm{U}^{35+}$ beam are listed in Table 2 for comparison.

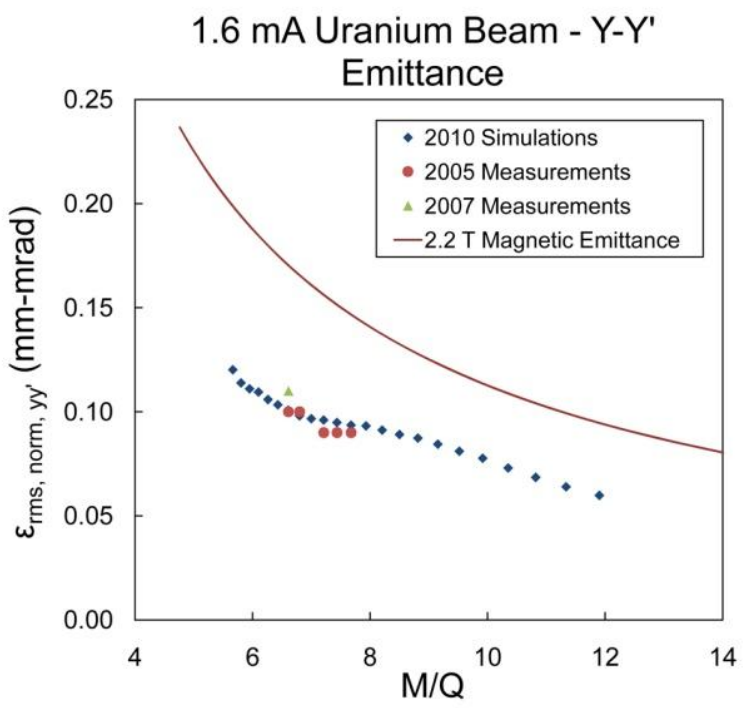

Figure 10: Comparison of emittances for $U^{20+}$ to $U^{43+}$ obtained by simulations and by experiment. Simulations were run with $80 \%$ neutralization and $1.6 \mathrm{~mA}$ total extracted current. 
Table 2: Comparison of 2-rms beam sizes in horizontal $(x)$ and vertical (y) direction for simulation and measurement of a $U^{35+}$ beam.

\begin{tabular}{|c|c|c|c|c|c|}
\hline \multirow{2}{*}{$\begin{array}{c}\text { Size } \\
(\mathrm{mm})\end{array}$} & \multicolumn{4}{|c|}{ Simulation } & \multirow{2}{*}{ Measured } \\
\cline { 2 - 5 } & $00 \%$ & $70 \%$ & $80 \%$ & $90 \%$ & \\
\hline$\Delta \mathrm{x}$ & 63.8 & 27.1 & 22.4 & 21.8 & 12.6 \\
$\Delta \mathrm{y}$ & 72.8 & 24.8 & 19.3 & 19.8 & 14.9 \\
\hline
\end{tabular}

\section{Comparison to emittance measurements.}

Figure 10 shows horizontal and vertical emittances from the simulation compared to measurements. The experimental data were taken with Allison type emittance scanners [16] at the position labeled "Diagnostics Box", seen in Figure 2. In 2005 the emittance scanners were positioned $2.767 \mathrm{~m}$ after extraction and in $20073.325 \mathrm{~m}$. Simulation emittances were evaluated $3.3 \mathrm{~m}$ after extraction. However, comparison of simulated emittances for different positions from $2.7 \mathrm{~m}$ to $3.3 \mathrm{~m}$ showed no differences, which is due to the fact that this region is a pure drift.

A trend of increasing emittance for decreasing $\mathrm{m} / \mathrm{q}$ is clearly seen in both simulation and measurement. The overall higher emittances in simulation could be due to non accounted beam losses, the initial ion distribution and properties or the changing neutralization levels in the beam line. The influences of these parameter will be further investigated.

\section{CONCLUSION}

The status of the efforts to create an adaptable simulation code for beam extraction from an ECR ion source using WARP has been presented A method has been developed to simulate multispecies beams with more realistic initial particle distributions and a high number of species in 3D mode with a high grid resolution. Emittance values are reproduced within reasonable margins by the simulations. Future work will aim to better understand the physics leading to the initial conditions and beam neutralization.

\section{ACKNOWLEDGEMENTS}

This work was supported by the Director, Office for Energy Research, Office of High Energy and Nuclear Physics, Nuclear Physics Division of the U.S. Department of Energy under Contract DE-AC02-05CH11231.

\section{REFERENCES}

[1] C.M. Lyneis et al., New Results with the superconducting ECR Ion Source VENUS, RSI 75 (2004).

[2] D. Leitner et al., Next generation ECR ion sources: First results of the superconducting $28 \mathrm{GHz}$ ECRIS VENUS, Nuclear Instruments and Methods in Physics Research Section B 235, 486 (2005).

[3] R.C. Pardo et al., Heavy-Ion Beams Required for the RIA Accelerator, RSI 75, 1427 (2004).
[4] D. S. Todd et al., Comparison of particle-in-cell simulation with experiment for the transport system of the superconducting electron cyclotron resonance ion source VENUS RSI 77 (2006).

[5] David P. Grote et al., The WARP Code: Modeling High Intensity Ion Beams

in 16th intern. Workshop on ECR Ion Sources, edited by M. Leitner (AIP, Berkeley, CA, 2004).

[6] D. S. Todd et al., Simulation and beamline experiments for the superconducting electron cyclotron resonance ion source VENUS, (AIP, 2008), p. 02A316.

[7] M. Cavenago et al., Model of accumulation, multiionization, and extraction of ions in ECR ion sources, (AIP, 2002), pp. 537.

[8] T. Thuillier et al., High current beam transport with Phoenix $28 \mathrm{GHz}$ : experiment and simulation, in 16th International Workshop on ECR Ion Sources, edited by M. Leitner (AIP Conference Proceedings, Berkeley, CA, 2004), pp. 41.

[9] P. Spädtke et al., Prospects of ion beam extraction and transport simulations (invited), Review of Scientific Instruments 79, 02B716 (2008).

[10] M. A. Leitner, D. C. Wutte, and C. M. Lyneis, Design Of The Extraction System of the Superconducting Ecr Ion Source Venus, in Particle Accelerator Conference (PAC'01) (The American Physical Society, IEEE, Chicago, 2001).

[11] R. Becker, and W. B. Herrmannsfeldt, Igun - A program for the simulation of positive ion extraction including magnetic fields, (AIP, 1992), pp. 2756.

[12] INP, KOBRA3-INP (INP, Junkernstr. 99, 65205 Wiesbaden, Germany.

[13] D. Leitner et al., High intensity production of high and medium charge state uranium and other heavy ion beams with VENUS, (AIP, 2008), p. 02C710.

[14] D. S. Todd, D.Leitner, and M.Strohmeier, Low Energy Beam Diagnostics at the VENUS ECR Ion Source, in BIW 2008Tahoe City, CA, 2008).

[15] A. Septier, Focusing of charged particles (Academic Press,, New York, 1967), Vol. 2.

[16] P.W.Allison, J.D.Sherman, and D.B.Holtkamp, An Emittance Scanner for Intense Low-Energy Ion Beams, IEEE Transactions on Nuclear Science NS-30 (1983).

This work performed under the auspices of the U.S. DOE by Lawrence Livermore Laboratory under Contract DE-AC52-07NA27344. 